\title{
Effectiveness of a care bundle to reduce central line-associated bloodstream infections
}

\section{The central \\ line-associated \\ bloodstream \\ infections rate \\ ... decreased \\ from 2.2 \\ in the pre- \\ intervention}

period to 0.5

in the post-

intervention

period

\section{Damoon \\ Entesari-Tatafi \\ MBBS $^{1}$ Hospital Geelong (UHG) ICU has reported CLABSI rates to VICNISS.}

Neil Orford MBBS, FCICM, PGDipEcho²

Michael J Bailey

$\mathrm{PhD}, \mathrm{MSc}, \mathrm{BSc}^{3,4}$

Martina N

Chonghaile

FCICM, FCARSCI, MBBCh ${ }^{2}$

Jill Lamb-Jenkins

RN, Grad Dip Ed, MN²

Eugene Athan MBBS, FRACP, MPH²

1 University Hospital Geelong Geelong, VIC.

2 Barwon Health Geelong, VIC.

3 Monash University, Melbourne, VIC

4 Alfred Hospital Melbourne, VIC.

dentes@

barwonhealth.org.au

doi: 10.5694/mjal4.01644
C entral line-associated bloodstream infections (CLABSIs) morbidity, mortality and cost. ${ }^{1}$ About 4000 CLABSIs occur in Australian intensive care units (ICUs) each year, with an estimated nationwide cost of $\$ 36.26$ million and a mortality rate of $4 \%-20 \%{ }^{2,3}$ The importance placed on CLABSI and its prevention has prompted standardised monitoring for quality assurance and innovation of preventive strategies. ${ }^{1,4,5}$ Care bundles focused on improving line insertion procedure have proven successful overseas. ${ }^{1,6}$ Local implementation of a similar care bundle to that used overseas across New South Wales proved successful, and prompted the Australian and New Zealand Intensive Care Society CLABSI Prevention Project. ${ }^{78}$ Despite these interventions, CLABSI rates range from 0.9 to 3.6 per 1000 central line days. ${ }^{6,79-20}$

The Victorian Healthcare Associated Infection Surveillance System (VICNISS) collects standardised ICU CLABSI rates for the state of Victoria. ${ }^{21}$ Since 2006, the University

An elevated reported CLABSI rate at UHG in 2007 and 2008 (3.8 and 3.6, respectively, compared with the state average of 2.7 per 1000 central line days $)^{22}$ prompted development and introduction of a CLABSI prevention care bundle. Our care bundle used an effective line insertion procedure identified from previous studies, ${ }^{1,6,7}$ but also incorporated a novel maintenance procedure. In this article, we report the effectiveness of this care bundle in a tertiary ICU in Victoria.

\section{Methods}

We undertook a before-and-after study, retrospectively accessing the pre-intervention data, at an adult, tertiary, 19-bed ICU that admits medical, surgical and cardiac surgical patients. are an important source of

\section{Abstract}

Objective: To determine the effectiveness of a care bundle, with a novel line maintenance procedure, in reducing the rate of central line-associated bloodstream infection (CLABSI) in the intensive care unit (ICU).

Design, participants and setting: Before-and-after study using CLABSI data reported to the Victorian Healthcare Associated Infection Surveillance System (VICNISS), in adult patients admitted to a tertiary adult ICU in regional Victoria between 1 July 2006 and 30 June 2014. VICNISSreported CLABSI cases were reviewed for verification. An intervention was implemented in 2009.

Intervention: The care bundle introduced in 2009 included a previously established line insertion procedure and a novel line maintenance procedure comprising Biopatch, daily $2 \%$ chlorhexidine body wash, daily ICU central line review, and liaison nurse follow-up of central lines.

Main outcome measures: CLABSI rate (cases per 1000 central line days).

Results: The average CLABSI rate fell from 2.2/1000 central line days (peak of $5.2 / 1000$ central line days in quarter 4,2008 ) during the pre-intervention period to $0.5 / 1000$ central line days ( $0 / 1000$ central line days from July 2012 to July 2014) during the post-intervention period.

Conclusion: Our study suggests that this care bundle, using a novel maintenance procedure, can effectively reduce the CLABSI rate and maintain it at zero out to 2 years.

Ethics approval was obtained from the Barwon Health Research Review Committee. This project was performed as part of the authors' usual roles and no funding or subsidy was received. All of us had full access to the study data.

\section{Intervention}

The care bundle was based on the Australian and New Zealand Intensive Care Society CLABSI prevention project, ${ }^{8}$ comprehensive literature review and collaboration between UHG ICU, UHG Infection Control Services and other key stakeholders. The final care bundle (Appendix 1) included standard line insertion procedure consistent with that described previously, ${ }^{6,7}$ bedside audit by an observer with stopping rules, and a novel line maintenance procedure that included placement of a Biopatch (Johnson and Johnson), sterile line access, daily $2 \%$ chlorhexidine body wash, daily central venous catheter (CVC) review with early line removal, and liaison nurse follow-up of all CVCs present at discharge.

\section{Study procedure}

All adult patients admitted to UHG ICU between 1 July 2006 and 30 June 2014 were captured in this study. The care bundle was introduced in 2009 , dividing patients into a preintervention period (1 July 2006 to 31 December 2009) and a post-intervention period (1 January 2010 to 30 June 2014). Case identification of CLABSI was based on the VICNISS dataset and review of blood cultures. All VICNISS-reported CLABSI cases were reviewed by one of us (DE) to confirm that they fulfilled the current VICNISS definition (Appendix 2). This definition is consistent with the internationally accepted O'Grady definition that has been previously applied. ${ }^{7,23}$

All confirmed CLABSIs were included in the analysis, irrespective of whether line insertion occurred in the ICU. Cohort demographic, basic clinical and microbiological data were collected from the hospital electronic database. Patient medical records of all VICNISS-reported CLABSI cases were reviewed to confirm CLABSI definition and collect additional clinical information. Finally, all positive 
1 Patient population characteristics, and ICU interventions and outcomes, for the pre- and post-intervention periods

Post-

\begin{tabular}{|c|c|c|c|}
\hline & Pre-intervention & intervention & $P$ \\
\hline No. & 4701 & 6273 & \\
\hline Mean age in years (SD) & $64.2(16.6)$ & $59.4(21.2)$ & $<0.001$ \\
\hline Male, no. (\%) & $2870(61 \%)$ & 3857 (61\%) & 0.64 \\
\hline Median APACHE III score (IQR) & $48(37-64)$ & $50(38-67)$ & 0.001 \\
\hline \multicolumn{4}{|l|}{ Comorbidity, no. (\%) } \\
\hline Respiratory & $233(5 \%)$ & $204(3 \%)$ & $<0.001$ \\
\hline Cardiovascular & $453(10 \%)$ & $176(3 \%)$ & $<0.001$ \\
\hline Hepatic & $42(1 \%)$ & $117(2 \%)$ & $<0.001$ \\
\hline Renal & $103(2 \%)$ & $134(2 \%)$ & 0.84 \\
\hline Immunosuppression & $271(6 \%)$ & $544(9 \%)$ & $<0.001$ \\
\hline Cancer & $231(5 \%)$ & $301(5 \%)$ & 0.78 \\
\hline \multicolumn{4}{|l|}{ Category, no. (\%) } \\
\hline Medical & $1863(40 \%)$ & $3250(52 \%)$ & $<0.001$ \\
\hline Surgical & $1071(23 \%)$ & $1027(16 \%)$ & $<0.001$ \\
\hline Cardiac surgical & $1767(38 \%)$ & $1996(32 \%)$ & $<0.001$ \\
\hline \multicolumn{4}{|l|}{ ICU admission source, no. (\%) } \\
\hline Operating theatre & $2752(59 \%)$ & 2988 (48\%) & $<0.001$ \\
\hline Emergency department & $910(19 \%)$ & $1565(25 \%)$ & $<0.001$ \\
\hline Ward & $801(17 \%)$ & $1280(20 \%)$ & $<0.001$ \\
\hline Other ICU & $235(5 \%)$ & $439(7 \%)$ & $<0.001$ \\
\hline \multicolumn{4}{|l|}{ ICU outcomes } \\
\hline Mechanical ventilation, no. (\%) & $2014(43 \%)$ & $3223(51 \%)$ & $<0.001$ \\
\hline Median ICU stay in hours (IQR) & $41.2(22.3-65.9)$ & $41.7(21.7-73.3)$ & 0.01 \\
\hline Median hospital stay in days (IQR) & $9.9(5.8-18.9)$ & $9.0(5.1-16.6)$ & $<0.001$ \\
\hline ICU mortality, no. (\%) & $333(7 \%)$ & $423(7 \%)$ & 0.70 \\
\hline Hospital mortality, no. (\%) & $534(11 \%)$ & $672(11 \%)$ & 0.34 \\
\hline
\end{tabular}

APACHE = Acute Physiology and Chronic Health Evaluation. ICU = intensive care unit. $\mathrm{IQR}=$ interquartile range.

blood cultures were blindly and independently reviewed by an infectious diseases specialist to identify any missing CLABSI cases.

\section{Statistical analysis}

Data were analysed using SAS, version 9.4 (SAS Institute). All data were visually assessed for normality using histograms. The primary outcome (CLABSI events) was compared first as an overall comparison of proportions and presented as a relative risk with $95 \%$ confidence intervals and second as the number of CLABSI events per quarter using Poisson regression.

Comparisons of pre- and post-intervention periods were performed for categorical variables using $\chi^{2}$ tests for equal proportions and reported as numbers (\%). Normally distributed variables were compared using Student $t$ tests and reported as mean (SD), and non-normally distributed data were compared using Wilcoxon rank-sum tests and reported as median (interquartile range). A twosided $P$ of 0.05 was considered to be statistically significant.

\section{Results}

Patient cohort characteristics are detailed in Box 1. The post-intervention cohort was significantly younger (mean age, 59.4 years v 64.2 years; $P<0.001)$ with a higher mean illness severity score (Acute Physiology and Chronic Health Evaluation
[APACHE] III score, $50 \mathrm{v} 48 ; P=0.001$ ), an increased proportion of medical patients (3250/6273 [52\%] v 1863/4701 [40\%]; $P<0.001)$, an increased requirement for mechanical ventilation (3223/6273 [51\%] v 2014/4701 [43\%]; $P<0.001)$ and an increased admission source from the wards or emergency department. Although the clinical significance of the differences in age and APACHE score are questionable, when all differences are considered together, they favour an increased risk of CLABSI in the post-intervention cohort.

A total of 24783 central line days occurred between July 2006 and June 2014 (Box 2). Thirty cases of CLABSI were included in the analysis (eight did not satisfy CLABSI definition criteria and were excluded - seven pre-intervention and one post-intervention; Appendix 3). No CLABSI cases additional to VICNISSreported cases were identified. In the pre-intervention period, there were 9844 central line days and 22 cases of CLABSI, resulting in a CLABSI rate of $2.2 / 1000$ central line days. In the postintervention period, there were 14939 central line days and eight cases of CLABSI, resulting in a CLABSI rate of $0.5 / 1000$ central line days. This represents a rate ratio of $0.23(95 \%$ CI, 0.11-0.54; $P=0.005)$. The temporal change in CLABSI rates is shown in Appendix 3 , with a peak CLABSI rate of 5.2/1000 (4/766) central line days in quarter 4 of 2008, and a CLABSI rate of zero since June 2012. The difference in the quarterly CLABSI rate before and after the intervention was introduced was significant $(P<0.001)$, as was the difference in the number of quarters in which CLABSI rate was zero (pre-intervention, $3 / 14 \mathrm{v}$ postintervention, $12 / 18 ; P=0.01$ ).

The blood culture collection rate $(60.1$ [2827/4701] v 61.5 [3859/6273] per 100 patients) was similar in the pre- and post-intervention periods, while the positive culture rate significantly fell from $9.1 \%(258 / 2827)$ to $7.2 \%(279 / 3859)$ $(P=0.005)$ (Box 2). Characteristics of the confirmed CLABSI cases are presented in Box 3. The site of blood culture collection was similar between the two cohorts; however, no common skin commensals were isolated 
as a causative organism in the postintervention cohort.

\section{Discussion}

Our study describes a significant reduction in the CLABSI rate in a tertiary Australian Victorian ICU from a peak quarterly rate of 5.2 to zero after implementation of a care bundle that incorporated a novel line maintenance procedure. Overall, the CLABSI rate, per 1000 central line days, decreased from 2.2 in the preintervention period to 0.5 in the postintervention period. In real terms, the reduced CLABSI rate equates to 15 fewer cases of CLABSI for the post-intervention period with an estimated total reduction in ICU length of stay of 38 days, hospital length of stay of 113 days and resultant cost saving of about $\$ 210000$.

To our knowledge, this is the first time that a zero CLABSI rate has been achieved and sustained in an Australian ICU. Burrell and colleagues reported a CLABSI rate of 0.9/1000 central line days from several centres. ${ }^{7}$ Department of Health data from Western Australia have shown similarly low CLABSI rates, but their processes were not reported. $5,7,24$

The finding of clinical effectiveness after introduction of the care bundle suggests that the observed benefits are causally associated. It is plausible that the maintenance procedure was crucial in reducing CLABSI, given that zero CLABSI was achieved despite the inclusion of lines inserted outside the ICU. It remains possible that changes in the patient cohort or procedures relating to CLABSI surveillance could account for the observed changes. In particular, there were seven CLABSIs that did not meet definition criteria in the pre-intervention period compared with one in the post-intervention period, raising the possibility of previous overreporting. Otherwise, the identified post-intervention cohort changes when taken together are considered as predisposing to CLABSI. In addition, the central line days and blood cultures per patient do not

2 Summary of total ICU patient admission, central line, blood culture and CLABSI data for the pre- and post-intervention periods

\begin{tabular}{lcccc} 
& $\begin{array}{c}\text { Pre- } \\
\text { intervention }\end{array}$ & $\begin{array}{c}\text { Post- } \\
\text { intervention }\end{array}$ & $\begin{array}{c}\text { Rate ratio } \\
(95 \% \mathrm{Cl})\end{array}$ & $\boldsymbol{P}$ \\
\hline Total patient days & 8070 & 10899 & & \\
Total central line days & 9844 & 14939 & & \\
Central line days per patient days & 1.22 & 1.37 & & \\
Total blood cultures & 2827 & 3859 & & \\
Blood cultures per patient days & 0.35 & 0.36 & $1.01(0.97-1.05)$ & 0.59 \\
ICU bacteraemia, no. (\%) & $258(9.1 \%)$ & $279(7.2 \%)$ & $0.79(0.67-0.93)$ & 0.005 \\
CLABSI, no. & 22 & 8 & $0.23(0.11-0.54)$ & 0.005 \\
CLABSI rate per 1000 central line days & 2.2 & 0.5 & & \\
\hline
\end{tabular}

CLABSI = central line-associated bloodstream infection. ICU = intensive care unit.

support altered clinical practice as an explanation.

Our study's strengths include a large patient cohort with availability of population characteristics, a microbiological blood culture dataset, an independent review of all positive blood cultures, and the application of the current standard CLABSI definition across the entire study period. This reduces the likelihood that the observed change was driven by changes in non-infection control related clinical practices. This study is limited by a single-centre retrospective, observational design, limiting generalisability and the ability to establish causality. However, these limitations are largely comparable to prior similar studies. ${ }^{6,725}$ Other limitations included potential confounding from lines inserted outside the ICU and the absence of adherence data for the individual components of our line maintenance procedure to show actual change in clinical practice. However, in our experience, the care bundle has been embedded into routine and has markedly improved clinical practice.

In conclusion, our study suggests that a central line care bundle with this novel line maintenance procedure can effectively reduce the CLABSI rate to zero and that this zero CLABSI rate can be sustained. Validation of our study by other centres, especially if performed prospectively, would further support our findings.

Acknowledgements: We acknowledge Jill Low and Kaylene Styles for their expert infection control input in the development of the intervention bundle and for

\section{Characteristics of CLABSI cases for the pre- and post-intervention periods}

\begin{tabular}{|c|c|c|}
\hline $\begin{array}{l}\text { Characteristics of } \\
\text { infected lines }\end{array}$ & $\begin{array}{l}\text { Pre-intervention } \\
\quad(n=22)\end{array}$ & $\begin{array}{l}\text { Post-intervention } \\
\qquad(n=8)\end{array}$ \\
\hline \multicolumn{3}{|l|}{ Line type, no. (\%) } \\
\hline CVC & $21(95 \%)$ & $6(75 \%)$ \\
\hline Vascath & $7(32 \%)$ & $3(38 \%)$ \\
\hline PAC & $1(5 \%)$ & $1(13 \%)$ \\
\hline Other & $3(14 \%)$ & $2(25 \%)$ \\
\hline $\begin{array}{l}\text { Median dwell time, days } \\
\text { (IQR) }\end{array}$ & $6(5-8)$ & $5(4-6)$ \\
\hline Inserted in ICU, no. (\%) & $15(68 \%)$ & $7(88 \%)$ \\
\hline \multicolumn{3}{|l|}{ CLABSI organism, no. (\%) } \\
\hline $\begin{array}{l}\text { Staphylococcus } \\
\text { aureus }\end{array}$ & $6(27 \%)$ & $2(25 \%)$ \\
\hline $\begin{array}{l}\text { Staphylococcus } \\
\text { epidermidis }\end{array}$ & $6(27 \%)$ & 0 \\
\hline Enterobacter spp. & $1(5 \%)$ & $2(25 \%)$ \\
\hline Candida spp. & $6(27 \%)$ & $2(25 \%)$ \\
\hline Enterococcus & $4(18 \%)$ & $1(13 \%)$ \\
\hline Other & $2(9 \%)$ & $3(38 \%)$ \\
\hline \multicolumn{3}{|c|}{ Positive blood culture site, no. (\%) } \\
\hline Peripheral & $5(23 \%)$ & $2(25 \%)$ \\
\hline Arterial & $1(5 \%)$ & 0 \\
\hline Central & $8(36 \%)$ & $2(25 \%)$ \\
\hline Unknown & $19(86 \%)$ & $5(63 \%)$ \\
\hline
\end{tabular}

providing surveillance data across the life of the study. We acknowledge Peter Stow for his support and input into the development and implementation of the intervention bundle.

Competing interests: No relevant disclosures.

Received 24 Nov 2014, accepted 9 Feb 2015 .

References are available online at www.mja.com.au. 
1 Weeks KR, Goeschel CA, Cosgrove $\mathrm{SE}$, et al. Prevention of central lineassociated bloodstream infections: a journey toward eliminating preventable harm. Curr Infect Dis Rep 2011; 13: 343-349.

2 Halton KA, Cook D, Paterson DL, et al. Cost-effectiveness of a central venous catheter care bundle. PLOS One 2010; 5: pii: el2815.

3 Australian Council on Healthcare Standards. Intensive care unit (ICU) central line-associated bloodstream infection (CLABSI): percentage of adult ICU-associated centrally inserted central line-associated bloodstream infection (Cl-CLABSI), during the 6 month time period. ACHS clinical indicator users' manual 2012. Sydney: ACHS, 2012.

4 McLaws ML, Taylor PC. The Hospital Infection Standardised Surveillance (HISS) programme: analysis of a two-year pilot. J Hosp Infect 2003; 53 : 259-267.

5 McLaws ML, Burrell AR. Zero risk for central line-associated bloodstream infection: are we there yet? Crit Care Med 2012; 40: 388-393.

6 Pronovost P, Needham D, Berenholtz $S$, et al. An intervention to decrease catheter-related bloodstream infections in the ICU. N Engl J Med 2006; 355: 2725-2732.

7 Central Line Associated Bacteraemia in NSW Intensive Care Units (CLAB ICU) Collaborative; Burrell AR, McLaws M, Murgo M, et al. Aseptic insertion of central venous lines to reduce bacteraemia. Med J Aust 2011; 194: 583-587.

8 Australian and New Zealand Intensive Care Society Safety and Quality Committee. Central line insertion and maintenance guideline. Melbourne: ANZICS, 2012. http://www.clabsi.com. au/images/Documents/ANZICS\%20 Central\%20Line\%20Insertion\%20 \%20Maintenance\%20Guidelines\%20 2012_04\%20W97_03.doc (accessed Jan 2015).

9 Munoz-Prince LS, Dezfulian C, Wyckoff M, et al. Effectiveness of stepwise interventions targeted to decrease central catheter-associated bloodstream infections. Crit Care Med 2012; 40: 1464-1469.

10 Timsit JF, Schwebel C, Bouadma L, et al. Chlorhexidine-impregnated sponges and less frequent dressing changes for prevention of catheterrelated infections in critically ill adults: a randomized controlled trial. JAMA 2009; 301: 1231-1241.

1 Timsit JF, Bouadma L, Ruckly S, et al. Dressing disruption is a major risk factor for catheter-related infections. Crit Care Med 2012; 40: 1707-1714.

12 Timsit JF, Mimoz O, Mourvillier B, et al. Randomized controlled trial of chlorhexidine dressing and highly adhesive dressing for preventing catheter-related infections in critically ill adults. Am J Respir Crit Care Med 2012; 186: 1272-1278.

13 Huang SS, Septimus E, Kleinman $\mathrm{K}$, et al. Targeted versus universal decolonization to prevent ICU infection. NEngl J Med 2013; 368: 2255-2265.

14 Marsteller JA, Sexton JB, Hsu YJ, et al. A multicenter, phased, clusterrandomized controlled trial to reduce central line-associated bloodstream infections in intensive care units. Crit Care Med 2012; 40: 2933-2939.

15 Derde LP, Dautzenberg MJ, Bonten MJ. Chlorhexidine body washing to control antimicrobial-resistant bacteria in intensive care units: a systematic review. Intensive Care Med 2012; 38 : 931-939.

16 Arvaniti K, Lathyris D, Clouva-Molyvdas $P$, et al. Comparison of Oligon catheters and chlorhexidine-impregnated sponges with standard multilumen central venous catheters for prevention of associated colonization and infections in intensive care unit patients: a multicenter, randomized, controlled study. Crit Care Med 2012; 40 : 420-429.

17 Ramos ER, Reitzel R, Jian Y, et al. Clinical effectiveness and risk of emerging resistance associated with prolonged use of antibioticimpregnated catheters: more than 0.5 million catheter days and 7 years of clinical experience. Crit Care Med 2011; 39: 245-251.
18 Cardo D, Dennehy PH, Halverson P, et al. Moving toward elimination of healthcare-associated infections: a call to action. Am J Infect Control 2010; 38: 671-675.

19 Walz JM, Avelar RL, Longtine KJ, et al. Anti-infective external coating of central venous catheters: a randomized, noninferiority trial comparing 5-fluorouracil with chlorhexidine/silver sulfadiazine in preventing catheter colonization. Crit Care Med 2010; 38: 2095-2102.

20 Chittick P, Sherertz RJ. Recognition and prevention of nosocomial vascular device and related bloodstream infections in the intensive care unit. Crit Care Med 2010; 38 (8 Suppl): S363-S372.

21 Russo PL, Bull A, Bennett N, et al. The establishment of a statewide surveillance program for hospitalacquired infections in large Victorian public hospitals: a report from the VICNISS Coordinating Centre. Am J Infect Control 2006; 34: 430-436.

22 Richards M, Dunkley C, Bull A, et al. VICNISS hospital acquired infection surveillance annual report 2008-09. Melbourne: Victorian Government Department of Health, 2010. http:// www.vicniss.org.au/Resources/ VICNISSAnnualReport2008-09.pdf (accessed Jan 2015).

23 O'Grady NP, Alexander M, Dellinger $E P$, et al. Guidelines for the prevention of intravascular catheter-related infections. Centers for Disease Control and Prevention. MMWR Recomm Rep 2002; 51: 1-29.

24 McCann R, Peterson A, Tempone S; Healthcare Associated Infection Unit. Healthcare infection surveillance Western Australia annual report 2012-2013. Perth: Department of Health Western Australia, 2013. http://www.public.health.wa.gov.au/ cproot/5796/2/hiswa-2012-13-ar-final. pdf (accessed Jan 2015).

25 Berenholtz SM, Pronovost PJ, Lipsett $P A$, et al. Eliminating catheter-related bloodstream infections in the intensive care unit. Crit Care Med 2004; 32: 2014-2020. 